\title{
Critical Higher Order Gravities in Higher Dimensions
}

\author{
Nahomi Kan* \\ Gifu National College of Technology, Motosu-shi, Gifu 501-0495, Japan \\ Koichiro Kobayashi ${ }^{\dagger}$ and Kiyoshi Shiraishi ${ }^{\ddagger}$ \\ Yamaguchi University, Yamaguchi-shi, Yamaguchi 753-8512, Japan
}

(Dated: November 10, 2018)

\begin{abstract}
We show that the higher order gravity model proposed by Meissner and Olechowski has a graviton mode, a massive spin-two excitation and no scalar mode in a maximally symmetric spacetime; therefore, by choosing the coefficients, we can construct a Lagrangian for 'critical gravity' from higher order terms of curvatures in higher dimensions. We also give a comment on construction of the theory with multi-criticality in higher order gravities.

PACS numbers: 04.50.-h, 04.50.Kd.
\end{abstract}

\footnotetext{
* kan@gifu-nct.ac.jp

$\dagger$ m004wa@yamaguchi-u.ac.jp

$\ddagger$ shiraish@yamaguchi-u.ac.jp
} 


\section{INTRODUCTION}

Effective theory of gravity may contain higher-derivative terms in its Lagrangian density, in addition to the Einstein-Hilbert term. The correction of the gravitational Lagrangian is motivated from studies on quantum theory of gravitation and string theory. Some special forms of higher order terms attract much attention nowadays.

In $D$-dimensional Lovelock gravity, whose the Lagrangian is written in the dimensionally continued Euler densities [1-9], the second derivative of the metric tensor disappears in the action. The excitation mode in the Lovelock gravity is only a (traceless, transverse) graviton mode in the $D$-dimensional Einstein background metric, as has recently been shown by Camanho, Edelstein and Paulos [10], Camanho and Edelstein [11], and Şişman, Güllü and Tekin [12].

On the other hand, Meissner and Olechowski have found the particular higher order invariants of the Ricci tensors and scalar curvatures which effectively coincide with the dimensionally continued Euler form if the spacetime is conformally flat $[13,14]$. Therefore, gravity theory governed by the Lagrangian constructed from a linear combination of such invariants has no scalar modes as the Einstein gravity.

In new massive gravity in three dimensions $[15,16]$ and critical gravity theory recently proposed in [17-22], scalar modes are absent from the particle content of the theory. In the present paper, we generalize the structure of the Lagrangian of the critical gravity to models with higher order terms in curvature tensors in $D$ dimensions. We show that such an extension can be attained by use of the curvature polynomials introduced by Meissner and Olechowski.

The outline of this paper is as follows. In $\S 2$, we construct the higher order term in curvatures by generalizing that in the Lagrangian of the critical gravity. In $\S 3$, The metric fluctuation on a maximally symmetric spacetime is analyzed in the special class of higher order gravity. We propose an extension of the critical gravity to more higher-derivative theory in $\S 4$. The final section is devoted to conclusion, where further prospect is also addressed. 


\section{LOVELOCK AND MEISSNER-OLECHOWSKI GRAVITY}

We shall begin with introducing the dimensionally continued Euler density:

$$
L_{L}^{(n)}=2^{-n} \delta_{\lambda_{1} \rho_{1} \cdots \lambda_{n} \rho_{n}}^{\sigma_{1} \tau_{1} \cdots \sigma_{n} \tau_{n}} R_{\sigma_{1} \tau_{1}}^{\lambda_{1} \rho_{1}} \cdots R^{\lambda_{n} \rho_{n}}{ }_{\sigma_{n} \tau_{n}}
$$

where $R_{\alpha \beta}^{\mu \nu}$ is the Riemann tensor and the generalized Kronecker delta is defined as

$$
\delta_{\nu_{1} \nu_{2} \cdots \nu_{p}}^{\mu_{1} \mu_{2} \cdots \mu_{p}} \equiv\left|\begin{array}{cccc}
\delta_{\nu_{1}}^{\mu_{1}} & \delta_{\nu_{2}}^{\mu_{1}} & \cdots & \delta_{\nu_{p}}^{\mu_{1}} \\
\delta_{\nu_{1}}^{\mu_{2}} & \delta_{\nu_{2}}^{\mu_{2}} & \cdots & \delta_{\nu_{p}}^{\mu_{2}} \\
\vdots & \vdots & \ddots & \vdots \\
\delta_{\nu_{1}}^{\mu_{p}} & \delta_{\nu_{2}}^{\mu_{p}} & \cdots & \delta_{\nu_{p}}^{\mu_{p}}
\end{array}\right| .
$$

One should note that

$$
\delta_{\mu \nu_{1} \nu_{2} \cdots \nu_{p}}^{\mu \mu_{1} \mu_{2} \cdots \mu_{p}}=(D-p) \delta_{\nu_{1} \nu_{2} \cdots \nu_{p}}^{\mu_{1} \mu_{2} \cdots \mu_{p}}
$$

where $D$ denotes the dimension of the spacetime; namely, all the indices take $\mu, \nu, \ldots=$ $0,1, \ldots, D-1$. The dimensionally continued Euler density $L_{L}^{(n)}$ consists of $n$-th order in the curvature tensors. For example, for $n=1$, we find the Einstein-Hilbert term

$$
L_{L}^{(1)}=R,
$$

where $R$ is the scalar curvature, and for $n=2$, we find the Gauss-Bonnet term

$$
L_{L}^{(2)}=R_{\mu \nu \rho \sigma} R^{\mu \nu \rho \sigma}-4 R_{\mu \nu} R^{\mu \nu}+R^{2},
$$

where $R_{\mu \nu}$ is the Ricci tensor. The Lovelock gravity is described by the Lagrangian density of a linear combination of the dimensionally continued Euler forms [2-4, 6-9].

Next, we consider the Schouten tensor (in our definition, which is different from the original definition by a factor $(D-2)^{-1}$,) which is known as

$$
S^{\mu \nu}=R^{\mu \nu}-\frac{1}{2(D-1)} R g^{\mu \nu} .
$$

The $n$-th order Meissner-Olechowski density is defined here by using the Schouten tensor and the generalized Kronecker delta as [13, 14, 23]

$$
L_{M O}^{(n)}=-\delta_{\nu_{1} \cdots \nu_{n}}^{\mu_{1} \cdots \mu_{n}} S_{\mu_{1}}^{\nu_{1}} \cdots S_{\mu_{n}}^{\nu_{n}}
$$

In the present paper, we call the theory governed by the Lagrangian density of a linear combination of this type as the Meissner-Olechowski gravity. 
Particularly, for $n=2$, we find the following quadratic combination in curvatures:

$$
L_{M O}^{(2)}=\left[R^{\mu \nu}-\frac{1}{2(D-1)} R g^{\mu \nu}\right]\left(R_{\mu \nu}-\frac{1}{2} R g_{\mu \nu}\right)=R_{\mu \nu} R^{\mu \nu}-\frac{D}{4(D-1)} R^{2}
$$

This scalar invariant is adopted in the argument on critical gravities in three and four dimensions [15-22]. Thus, the Meissner-Olechowski gravity is a possible candidate for an extension of the critical gravity.

\section{ANALYSIS ON METRIC FLUCTUATION}

We consider perturbative metric fluctuation around a static, curved background in this section. One should find the linearized gravitational field equation in order to identify the particle content in the theory. Assuming that the metric is divided into the background and fluctuation, we write

$$
g_{\mu \nu}=\bar{g}_{\mu \nu}+h_{\mu \nu}
$$

The indices are raised and lowered by the background metric $\bar{g}$. Then the trace of the fluctuation is expressed as

$$
h \equiv \bar{g}^{\mu \nu} h_{\mu \nu} .
$$

The following expansions to the quadratic order in $h_{\mu \nu}$ are generally known [24]:

$$
\begin{gathered}
\int d^{D} x \sqrt{-g}=\int d^{D} x \sqrt{-\bar{g}}\left[1+\frac{1}{2} h+\frac{1}{8}\left(h^{2}-2 h^{\mu \nu} h_{\mu \nu}\right)+O\left(h^{3}\right)\right] \\
\int d^{D} x \sqrt{-g} R=\int d^{D} x \sqrt{-\bar{g}}\left(\frac{1}{2} \bar{R} \bar{g}^{\mu \nu}-\bar{R}^{\mu \nu}\right) h_{\mu \nu} \\
+\int d^{D} x \sqrt{-\bar{g}}\left[\frac{1}{4} h^{\mu \nu} \bar{\nabla}^{2} h_{\mu \nu}+\frac{1}{2} h \bar{\nabla}_{\mu} \bar{\nabla}_{\nu} h^{\mu \nu}-\frac{1}{2} h_{\mu \nu} \bar{\nabla}^{\mu} \bar{\nabla}_{\lambda} h^{\lambda \nu}-\frac{1}{4} h \bar{\nabla}^{2} h+\frac{1}{2} h^{\mu \nu} \bar{R}_{\mu}^{\alpha}{ }_{\nu}^{\beta} h_{\alpha \beta}\right. \\
\left.+\frac{1}{2} h^{\mu \nu} \bar{R}_{\mu}^{\alpha} h_{\alpha \nu}-\frac{1}{2} h \bar{R}^{\mu \nu} h_{\mu \nu}-\frac{1}{4} \bar{R} h^{\mu \nu} h_{\mu \nu}+\frac{1}{8} \bar{R} h^{2}+O\left(h^{3}\right)\right],
\end{gathered}
$$

where $\bar{R}_{\mu \nu \lambda \rho}, \bar{R}_{\mu \nu}$, and $\bar{R}$ are the Riemann tensor, the Ricci tensor and the scalar curvature derived only from the background metric $\bar{g}_{\mu \nu}$.

Now, we consider a $D$-dimensional maximally symmetric spacetime as the background. Then, the Riemann tensor can be written as

$$
\bar{R}_{\alpha \beta}^{\mu \nu}=\Lambda\left(\delta_{\alpha}^{\mu} \delta_{\beta}^{\nu}-\delta_{\beta}^{\mu} \delta_{\alpha}^{\nu}\right)
$$


where $\Lambda$ is a constant. This background geometry is the solution of the Einstein equation obtained by varying the action

$$
S_{0}(\Lambda)=\int d^{D} x \sqrt{-g}[R-(D-1)(D-2) \Lambda] .
$$

The Riemann tensor, the Ricci tensor and the scalar curvature are written to the first order in $h_{\mu \nu}$ as

$$
R_{\alpha \beta}^{\mu \nu}=\bar{R}_{\alpha \beta}^{\mu \nu}+\mathcal{R}_{\alpha \beta}^{\mu \nu}+O\left(h^{2}\right), \quad R_{\alpha}^{\mu}=\bar{R}_{\alpha}^{\mu}+\mathcal{R}_{\alpha}^{\mu}+O\left(h^{2}\right), \quad R=\bar{R}+\mathcal{R}+O\left(h^{2}\right),
$$

(here and hereafter, the abbreviation such that $O(h)=O\left(h_{\mu \nu}\right)$ is used), where

$$
\begin{aligned}
\mathcal{R}_{\alpha \beta}^{\mu \nu}= & -\frac{1}{2} \Lambda\left(\delta_{\alpha}^{\mu} h_{\beta}^{\nu}-\delta_{\beta}^{\mu} h_{\alpha}^{\nu}-\delta_{\alpha}^{\nu} h_{\beta}^{\mu}+\delta_{\beta}^{\nu} h_{\alpha}^{\mu}\right) \\
& +\frac{1}{2}\left(-\bar{\nabla}_{\alpha} \bar{\nabla}^{\mu} h_{\beta}^{\nu}+\bar{\nabla}_{\alpha} \bar{\nabla}^{\nu} h_{\beta}^{\mu}+\bar{\nabla}_{\beta} \bar{\nabla}^{\mu} h_{\alpha}^{\nu}-\bar{\nabla}_{\beta} \bar{\nabla}^{\nu} h_{\alpha}^{\mu}\right), \\
\mathcal{R}_{\alpha}^{\mu}= & \Lambda\left(h_{\alpha}^{\mu}-\delta_{\alpha}^{\mu} h\right)+\frac{1}{2}\left(-\bar{\nabla}^{2} h_{\alpha}^{\mu}-\bar{\nabla}_{\alpha} \bar{\nabla}^{\mu} h+\bar{\nabla}^{\mu} \bar{\nabla}_{\nu} h_{\alpha}^{\nu}+\bar{\nabla}_{\alpha} \bar{\nabla}_{\nu} h^{\nu \mu}\right), \\
\mathcal{R}= & -(D-1) \Lambda h+\bar{\nabla}_{\mu} \bar{\nabla}_{\nu} h^{\mu \nu}-\bar{\nabla}^{2} h .
\end{aligned}
$$

The Schouten tensor introduced in the previous section is also expanded by the order of $h_{\mu \nu}$ around the background. We define the fluctuated part of the Schouten tensor as

$$
S_{\Lambda_{\alpha}^{\mu}}^{\mu} \equiv S_{\alpha}^{\mu}-\bar{S}_{\alpha}^{\mu}=R_{\alpha}^{\mu}-\frac{1}{2(D-1)} R \delta_{\alpha}^{\mu}-\frac{D-2}{2} \Lambda \delta_{\alpha}^{\mu}=\mathcal{R}_{\alpha}^{\mu}-\frac{1}{2(D-1)} \mathcal{R} \delta_{\alpha}^{\mu}+O\left(h^{2}\right),
$$

where the barred quantities indicates that it is estimated in the background metric, or, the quantity in $O\left(h^{0}\right)$. Using this tensor instead of the 'whole' Schouten tensor, we find that the second order Meissner-Olechowski density becomes

$$
\begin{aligned}
L_{M O \Lambda}^{(2)} & \equiv-\delta_{\mu \nu}^{\alpha \beta} S_{\Lambda_{\alpha}^{\mu}}^{\mu} S_{\Lambda}^{\nu} \\
& =\left[R_{\alpha}^{\mu}-\frac{1}{2(D-1)} R \delta_{\alpha}^{\mu}-\frac{D-2}{2} \Lambda \delta_{\alpha}^{\mu}\right]\left[R_{\mu}^{\alpha}-\frac{1}{2} R \delta_{\mu}^{\alpha}+\frac{(D-1)(D-2)}{2} \Lambda \delta_{\mu}^{\alpha}\right] \\
& =L_{M O}^{(2)}+\frac{(D-2)^{2}}{2} \Lambda R-\frac{D(D-1)(D-2)^{2}}{4} \Lambda^{2} .
\end{aligned}
$$

On the other hand, since $S_{\Lambda_{\alpha}^{\mu}}^{\mu}$ is obviously of linear and higher order in $h_{\mu \nu}$, it is clear that

$$
L_{M O \Lambda}^{(2)}=\mathcal{R}_{\alpha}^{\mu} \mathcal{R}_{\mu}^{\alpha}-\frac{D}{4(D-1)} \mathcal{R}^{2}+O\left(h^{3}\right),
$$

i.e., $\mathcal{R}_{\alpha}^{\mu} \mathcal{R}_{\mu}^{\alpha}-\frac{D}{4(D-1)} \mathcal{R}^{2}$ is the quantity of $O\left(h^{2}\right)$. The explicit calculation using (3.9) and (3.10) yields

$$
\mathcal{R}_{\alpha}^{\mu} \mathcal{R}_{\mu}^{\alpha}-\frac{D}{4(D-1)} \mathcal{R}^{2} \simeq \Lambda^{2}\left[h_{\alpha}^{\mu} h_{\mu}^{\alpha}+(D-2) h^{2}-\frac{1}{4} D(D-1) h^{2}\right]-\frac{D-3}{4} \Lambda h \bar{\nabla}^{2} h
$$




$$
\begin{aligned}
& -\Lambda h_{\mu}^{\alpha} \bar{\nabla}^{2} h_{\alpha}^{\mu}-(D-1) \Lambda h_{\mu}^{\alpha} \bar{\nabla}^{\mu} \bar{\nabla}_{\nu} h_{\alpha}^{\nu}+\frac{D}{2} \Lambda h \bar{\nabla}_{\mu} \bar{\nabla}_{\nu} h^{\mu \nu} \\
& +\frac{1}{4}\left[h_{\mu}^{\alpha} \bar{\nabla}^{2} \bar{\nabla}^{2} h_{\alpha}^{\mu}-\left(\bar{\nabla}_{\mu} \bar{\nabla}_{\nu} h^{\mu \nu}\right)^{2}-\frac{1}{D-1}\left(\bar{\nabla}_{\mu} \bar{\nabla}_{\nu} h^{\mu \nu}-\bar{\nabla}^{2} h\right)^{2}\right. \\
& \left.-\left(\bar{\nabla}^{\mu} \bar{\nabla}_{\nu} h_{\alpha}^{\nu}-\bar{\nabla}_{\alpha} \bar{\nabla}_{\nu} h^{\nu \mu}\right)^{2}\right]
\end{aligned}
$$

where ' $\simeq$ ' denotes the equality up to the total derivatives.

Now, let us suppose the action

$$
S=S_{0}(\Lambda)+\alpha \int d^{D} x \sqrt{-g} L_{M O \Lambda}^{(2)}
$$

We can analyze the linearized equation of motion for $h_{\mu \nu}$ by taking the variation of the action up to $O\left(h^{2}\right)$. Applying the following gauge choice

$$
\bar{\nabla}_{\nu} h^{\nu \mu}-\bar{\nabla}^{\mu} h=0
$$

one can find the trace of the equation of motion derived from the action (3.15) leads to

$$
h=0 \text {. }
$$

Therefore we get the following on-shell equation of motion for the metric fluctuation, which is transverse and traceless $\left(\bar{\nabla}_{\mu} h^{\mu \nu}=h=0\right)$, on the maximally symmetric spacetime:

$$
\alpha\left(\bar{\nabla}^{2}-2 \Lambda\right)\left(\bar{\nabla}^{2}-2 \Lambda+\frac{1}{\alpha}\right) h_{\mu \nu}=0 .
$$

Note that the action $(3.15)$ can be rewritten as

$$
\begin{aligned}
S_{0}(\Lambda)+\alpha \int d^{D} x \sqrt{-g} L_{M O \Lambda}^{(2)} \\
=\int d^{D} x \sqrt{-g}\left[\left\{1+\frac{(D-2)^{2}}{2} \alpha \Lambda\right\} R-(D-1)(D-2)\left\{1+\frac{D(D-2)}{4} \alpha \Lambda\right\} \Lambda\right. \\
\left.+\alpha L_{M O}^{(2)}\right],
\end{aligned}
$$

and this is just the action considered in the original literature of critical gravity, after rescaling the Newton and cosmological constants.

Eq. (3.18) indicates that there are two tensor excitation modes in general cases [17-22]. Furthermore, one can find that the critical point exists at $\alpha \rightarrow \infty$, or equivalently, when the action then becomes $\int d^{D} x \sqrt{-g} L_{M O \Lambda}^{(2)}$. At this point, a single graviton excitation appears but it exists a log mode $[25-27]^{1}$.

\footnotetext{
${ }^{1}$ The first work on the log mode, in topologically massive gravity in three dimension, has been done by Grumiller and Johansson [28]. For a recent review, see Ref. [29].
} 
The argument so far is not new one but a lightning review of critical gravity. We turn to generalize the construction of critical gravity by using higher order terms in curvatures. We consider the following higher order scalar invariants:

$$
\begin{aligned}
L_{M O}^{(n+2)}{ }_{\Lambda} & \equiv-\delta_{\nu_{1} \cdots \nu_{n} \mu \nu}^{\mu_{1} \cdots \mu_{n} \alpha \beta} S_{\mu_{1}}^{\nu_{1}} \cdots S_{\mu_{n}}^{\nu_{n}} S_{\Lambda_{\alpha}}^{\mu} S_{\Lambda_{\beta}}^{\nu} \\
& =-\delta_{\nu_{1} \cdots \nu_{n} \mu \nu}^{\mu_{1} \cdots \mu_{n} \alpha \beta}\left(\bar{R}_{\mu_{1}}^{\nu_{1}}-\frac{1}{2(D-1)} \bar{R} g_{\mu_{1}}^{\nu_{1}}\right) \cdots\left(\bar{R}_{\mu_{n}}^{\nu_{n}}-\frac{1}{2(D-1)} \bar{R} g_{\mu_{n}}^{\nu_{n}}\right) S_{\Lambda_{\alpha}}^{\mu} S_{\Lambda_{\beta}}^{\nu}+O\left(h^{3}\right) \\
& =-(D-2)(D-3) \cdots(D-n-1)\left(\frac{D-2}{2} \Lambda\right)^{n} \delta_{\mu \nu}^{\alpha \beta} S_{\Lambda_{\alpha}}^{\mu} S_{\Lambda_{\beta}}^{\nu}+O\left(h^{3}\right) \\
& =(D-2)(D-3) \cdots(D-n-1)\left(\frac{D-2}{2} \Lambda\right)^{n} L_{M O \Lambda}^{(2)}+O\left(h^{3}\right) \\
& =\frac{(D-2) !}{(D-n-2) !}\left(\frac{D-2}{2} \Lambda\right)^{n}\left[\mathcal{R}_{\alpha}^{\mu} \mathcal{R}_{\mu}^{\alpha}-\frac{D}{4(D-1)} \mathcal{R}^{2}\right]+O\left(h^{3}\right) .
\end{aligned}
$$

Thus, this is trivially of $O\left(h^{2}\right)$. On the other hand, we find the equality:

$$
\begin{aligned}
& L_{M O}^{(n+2)}{ }_{\Lambda}=-\delta_{\nu_{1} \cdots \nu_{n} \mu \nu}^{\mu_{1} \cdots \mu_{n} \alpha \beta} S_{\mu_{1}}^{\nu_{1}} \cdots S_{\mu_{n}}^{\nu_{n}} S_{\Lambda_{\alpha}}^{\mu} S_{\Lambda_{\beta}}^{\nu} \\
= & L_{M O}^{(n+2)}-(D-n-1)(D-2) \Lambda L_{M O}^{(n+1)}+(D-n)(D-n-1)\left(\frac{D-2}{2}\right)^{2} \Lambda^{2} L_{M O}^{(n)}(3.2
\end{aligned}
$$

From (3.12) and (3.21), since $L_{M O}^{(1)} \propto R$ contains at most second derivatives of $h_{\mu \nu}$ and $L_{M O}^{(2)}$ contains at most fourth-order derivatives, we find that all $L_{M O}^{(n)}(n \geq 2)$ contains at most fourth-order derivative of the metric fluctuation on the maximally symmetric geometry. ${ }^{2}$ Moreover, because Eq. (3.20) shows that $L_{M O \Lambda}^{(n)}(n \geq 2)$ is proportional to $L_{M O \Lambda}^{(2)}$, the equation of motion can be reduced to the form similar to (3.18).

Solving the recursion relation (3.21) with Eq. (3.12), we obtain

$$
\begin{aligned}
L_{M O}^{(n)}=\frac{(D-2) !}{(D-n) !} & \left(\frac{D-2}{2}\right)^{n}\left\{-D(D-1) \Lambda^{n}-n \Lambda^{n-1}\left[\frac{D-2}{2} \Lambda h_{\mu \nu} h^{\mu \nu}+\frac{1}{4} h_{\mu \nu} \bar{\nabla}^{2} h^{\mu \nu}\right]\right. \\
& \left.+\frac{n(n-1)}{2} \Lambda^{n-2}\left[\Lambda^{2} h_{\mu \nu} h^{\mu \nu}-\Lambda h_{\mu \nu} \bar{\nabla}^{2} h^{\mu \nu}+\frac{1}{4} h_{\mu \nu} \bar{\nabla}^{2} \bar{\nabla}^{2} h^{\mu \nu}\right]\right\},
\end{aligned}
$$

where unphysical modes are discarded, and when the background geometry, as a solution of the equation of motion, is expressed by (3.5).

To summarize, the particle content of the the Meissner and Olechowski gravity governed by the action

$$
S=S_{0}\left(\Lambda^{\prime}\right)+\int d^{D} x \sqrt{-g} \sum_{n=2}^{D} \alpha_{n}^{\prime} L_{M O}^{(n)}
$$

\footnotetext{
${ }^{2}$ If we define $L_{M O}^{(1)}=-\frac{D-2}{2(D-1)} R$ and $L_{M O}^{(0)}=-1$, Eq. (3.21) holds for $n \geq 0$.
} 
includes two transverse traceless modes only, if $\Lambda^{\prime}$ is chosen so that the classical background solution arises as $\bar{R}_{\alpha \beta}^{\mu \nu}=\Lambda\left(\delta_{\alpha}^{\mu} \delta_{\beta}^{\nu}-\delta_{\beta}^{\mu} \delta_{\alpha}^{\nu}\right){ }^{3}$

In particular,

$$
S_{c r i t}=\int d^{D} x \sqrt{-g} \sum_{n=2}^{D} \alpha_{n} L_{M O \Lambda}^{(n)}
$$

yields the critical gravity. ${ }^{4}$ Therefore this is an extension of the critical gravity to higher order Lagrangian. It is important to note that since the generalized Kronecker delta is restricted by the number of dimensions, the Lagrangian (3.23) is at most $D$-th order in the curvatures.

\section{COMMENT ON MORE HIGHER ORDER GRAVITY AND MULTI-CRITICAL GRAVITY}

The order of the Meissner and Olechowski gravity is limited by the number of dimensions, i.e., it is valid for $n \leq D$. As is obvious at a glance, the Lagrangian density consisting of combinations of the type $f\left(R_{\mu \nu \rho \sigma}\right) L_{M O \Lambda}^{(2)}$ may lead to the critical gravity. Note, however, that it is very complicated to find the background metric, as the solution of the equation of motion derived from such a general class of higher order Lagrangian. Here, we wish to find higher-order, symmetric invariants of curvature tensors by systematic construction.

One should have noticed the beautiful symmetry is implemented in the Lovelock gravity. According to Ref. [10-12], the Lovelock gravity on a maximally symmetric spacetime contains a single graviton mode just as in the Einstein gravity. Therefore we find that the Lovelock tensor

$$
G_{\nu}^{(n)^{\mu}} \equiv-\frac{1}{\sqrt{-g}} \frac{\delta\left(\int d^{D} x \sqrt{-g} L_{L}^{(n)}\right)}{\delta g_{\rho \mu}} g_{\nu \rho}=-2^{-(n+1)} \delta_{\nu \lambda_{1} \rho_{1} \cdots \lambda_{n} \rho_{n}}^{\mu \sigma_{1} \tau_{1} \cdots \sigma_{n} \tau_{n}} R_{\sigma_{1} \tau_{1}}^{\lambda_{1} \rho_{1}} \cdots R_{\sigma_{n} \tau_{n}}^{\lambda_{n} \rho_{n}}
$$

is expanded in terms of the metric fluctuation $h_{\mu \nu}$ on the background spacetime, where the Riemann tensor is given by (3.5), as

$$
G_{\alpha}^{(m) \mu}-\bar{G}_{\alpha}^{(m) \mu} \propto \mathcal{R}_{\alpha}^{\mu}-\frac{1}{2} \mathcal{R} \delta_{\alpha}^{\mu}+O\left(h^{2}\right) .
$$

${ }^{3}$ If we introduce an auxiliary field $s_{\mu}^{\nu}, L_{M O}^{(n)}$ can be replaced by $-\delta_{\nu_{1} \cdots \nu_{n}}^{\mu_{1} \cdots \mu_{n}} s_{\mu_{1}}^{\nu_{1}} \cdots s_{\mu_{n}}^{\nu_{n}}-$ $n \delta_{\nu_{1} \cdots \nu_{n-1} \nu}^{\mu_{1} \cdots \mu_{n-1} \mu} s_{\mu_{1}}^{\nu_{1}} \cdots s_{\mu_{n-1}}^{\nu_{n-1}}\left(R_{\mu}^{\nu}-\frac{1}{2(D-1)} R \delta_{\mu}^{\nu}-s_{\mu}^{\nu}\right)$

${ }^{4}$ It is easily shown that the background (3.5) is the solution of the equation of motion derived from (3.24), by doing the explicit calculation as in Ref. [23]. 
We naturally define an extension of the Schouten tensor as

$$
S_{(m)}^{\mu \nu} \equiv G^{(m)^{\mu \nu}}-\frac{1}{D-1} G^{(m)} g^{\mu \nu},
$$

where $G^{(n)} \equiv G_{\rho}^{(n) \rho}$. Then the following relation is obvious:

$$
S_{\alpha}^{(m) \mu}-\bar{S}_{\alpha}^{(m) \mu} \propto \mathcal{R}_{\alpha}^{\mu}-\frac{1}{2(D-1)} \mathcal{R} \delta_{\alpha}^{\mu}+O\left(h^{2}\right) .
$$

It is now evident by the same argument in the previous section that the Lagrangian density consisting of a certain linear combination of the following terms

$$
L_{M O}^{(n \cdot m)}=-\delta_{\nu_{1} \cdots \nu_{n}}^{\mu_{1} \cdots \mu_{n}} S_{(m)}^{\nu_{\mu_{1}}} \cdots S_{(m)_{\mu_{n}}}^{\nu_{n}}
$$

leads to a model of critical gravity. ${ }^{5}$

The gravity theory with multi-critical points, or dubbed as 'polycritical' gravity, has been proposed in Refs. [30-34]. In the theory, the analysis on the equation of motion for the metric fluctuation reveals that there is no scalar mode and three or more spin-two excitation due to the higher derivatives than the sixth order. The generalization of the construction in Refs. [30-34] to the higher order gravity can be obtained by taking the following iterative consideration.

To obtain the equation of motion with sixth order derivatives for the metric fluctuation, we adopt the Lagrangian density

$$
L_{B}=K^{(n) \mu \nu} \Delta_{\mu \nu \rho \sigma} G^{(m) \rho \sigma},
$$

where

$$
K^{(n) \mu \nu} \equiv-\frac{1}{\sqrt{-g}} \frac{\delta \int d^{D} x \sqrt{-g} L_{M O}^{(n)}}{\delta g_{\mu \nu}},
$$

and

$$
\Delta_{\mu \nu \rho \sigma} \equiv \frac{1}{2}\left(g_{\mu \rho} g_{\nu \sigma}+g_{\mu \sigma} g_{\nu \rho}\right)-\frac{1}{D-1} g_{\mu \nu} g_{\rho \sigma} .
$$

Similarly, the eighth order differential equation for $h_{\mu \nu}$ is derived from

$$
L_{B}=K^{(n) \mu \nu} \Delta_{\mu \nu \rho \sigma} K^{(m) \rho \sigma} .
$$

\footnotetext{
${ }^{5}$ We should note that the auxiliary field method $[15,16,26]$ also leads to the term which can be used in critical higher order gravity, such as $-s_{(n)}{ }^{\mu \nu} G_{\mu \nu}^{(m)}-s_{(m)}{ }^{\mu \nu} G_{\mu \nu}^{(n)}+s_{(n)}^{\mu \nu} s_{(m) \mu \nu}-s_{(n) \rho} \rho_{(m) \rho}^{\rho}$, where $s_{(m)}^{\mu \nu}$ and $s_{(n)}^{\mu \nu}$ are auxiliary fields.
} 
To get more higher derivatives on the metric fluctuation, we repeat the iteration

$$
L_{N}=-\frac{1}{\sqrt{-g}} \frac{\delta \int d^{D} x \sqrt{-g} L_{B}}{\delta g_{\mu \nu}} \Delta_{\mu \nu \rho \sigma} G^{(m) \rho \sigma},
$$

or

$$
L_{N N}=-\frac{1}{\sqrt{-g}} \frac{\delta \int d^{D} x \sqrt{-g} L_{B}}{\delta g_{\mu \nu}} \Delta_{\mu \nu \rho \sigma} K^{(m) \rho \sigma},
$$

and obtain the Lagrangian which yields higher order differential equation for the metric fluctuation. It has been explicitly confirmed that there is no scalar mode in the theory governed by these types of the Lagrangian density by using conformally flat metrics in Ref. [23]. Therefore a certain linear combinations of these terms yields polycritical gravity, though the critical relation among the coefficients is not pursued here.

Finally, it is interesting to point out that these candidate Lagrangians have a form very akin to the Lagrangian with the 'detailed balance condition' of the Hořava-Lifshitz gravity $[35]$.

\section{SUMMARY AND CONCLUSION}

In the present paper, we have studied a certain models with higher order terms in curvatures, in which excitation modes are similar to ones in critical gravity. We have confirmed that the Lagrangian consisting of the Meissner-Olechowski densities yields the higher-derivative gravity without scalar modes and have found that the peculiar combination $L_{M O \Lambda}^{(n)}$ leads to the critical gravity with the fourth-order derivatives on the tensor mode. We have also given a discussion on the possible construction of terms which can be utilized in multi-critical gravity.

In general, higher order gravity possesses many solutions. For an interesting example, the Lagrangian consisting of a single Meissner-Olechowski density, $\int d^{D} x \sqrt{-g} L_{M O \Lambda}^{(n)}$, admits two distinct solutions, in which the curvatures are $\bar{R}_{\alpha \beta}^{\mu \nu}=\Lambda\left(\delta_{\alpha}^{\mu} \delta_{\beta}^{\nu}-\delta_{\alpha}^{\nu} \delta_{\beta}^{\mu}\right)$ and $\bar{R}_{\alpha \beta}^{\mu \nu}=$ $\frac{D-2 n}{D-2 n-4} \Lambda\left(\delta_{\alpha}^{\mu} \delta_{\beta}^{\nu}-\delta_{\alpha}^{\nu} \delta_{\beta}^{\mu}\right)$. Of course, a fluctuation mode on the latter background becomes massive and the theory on it is not critical gravity. The study of possible vacuum transition by using the massless, massive, and log modes on the vacua seems to be interesting, since the investigation may give a novel cosmological evolution.

An extension of the Randall-Sundrum models [36, 37], in which the spacetime is asymptotically anti-de Sitter geometry, can be considered by connecting the model with higher 
order gravity. In a general higher order gravity, however, a thin brane require more singularity than a usual delta function source, because of the existence of higher derivatives on the metric fluctuation. Only the Lovelock gravity has been applied to the Randall-Sundrum models thus so far. A possibility of higher order generalization is that one use two or more Meissner-Olechowski densities for the gravitational Lagrangian, in order to cancel the fourth-order derivative (thus this construction no longer leads to critical gravity!). Another possibility is to consider thick branes $[38,39]$. Also in this case, the scalar field configuration coupled to higher order gravity naturally leads to many vacua. Therefore, the study of thick wall in the cosmological context is also an interesting subject.

Because the Meissner-Olechowski gravity model does not contain the Riemann tensors, the supersymmetrization of the model is expected to be easy at on-shell condition in a perturbative approach. By the same reason, quantum correction in the naive sense may also be easy to handle in the model. Also, some compactifications and black hole solutions in the Meissner-Olechowski gravity are interesting and worth studying in asymptotically antide Sitter spacetimes. We shall return to these various aspects of the Meissner-Olechowski gravity in future work.

\section{ACKNOWLEDGMENTS}

The authors would like to thank T. Zojer for appropiate information on the work on the logarithmic modes. We also would like to thank S. Deser and J. Edelstein for suggestion of suitable references.

[1] C. Lanczos, Z. Phys. 73 (1932) 147; Annals Math. 39 (1938) 842.

[2] D. Lovelock, J. Math. Phys. 12 (1971) 498; J. Math. Phys. 13 (1972) 874.

[3] B. Zumino, Phys. Rep. 137 (1985) 109.

[4] B. Zwiebach, Phys. Lett. B156 (1985) 315.

[5] D. G. Boulware and S. Deser, Phys. Rev. Lett. 55 (1985) 2656.

[6] J. T. Wheeler, Nucl. Phys. B268 (1986) 737.

[7] C. Aragone, Phys. Lett. B186 (1987) 151.

[8] F. Müller-Hoissen, Phys. Lett. B163 (1985) 106; Class. Quant. Grav. 3 (1986) 665. 
[9] T. Padmanabhan and D. Kothawala, arXiv:1302.2151 [gr-qc]

[10] X. O. Camanho, J. D. Edelstein and M. F. Paulos, JHEP 1105 (2011) 127.

[11] X. O. Camanho and J. D. Edelstein, Class. Quant. Grav. 30 (2013) 035009.

[12] T. Ç. Şişman, İ. Güllü and B. Tekin, Phys. Rev. D86 (2012) 044041.

[13] K. A. Meissner and M. Olechowski, Phys. Rev. Lett. 86 (2001) 3708;

[14] K. A. Meissner and M. Olechowski, Phys. Rev. D65 (2002) 064017;

[15] E. A. Bergshoeff, O. Hohm and P. K. Townsend, Phys. Rev. Lett. 102 (2009) 201301.

[16] E. A. Bergshoeff, O. Hohm and P. K. Townsend, Phys. Rev. Lett. D79 (2009) 124042.

[17] İ. Güllü and B. Tekin, Phys. Rev. D80 (2009) 064033.

[18] H. Lü and C. N. Pope, Phys. Rev. Lett. 106 (2011) 181302.

[19] S. Deser, H. Liu, H. Lü, C. N. Pope, T. Ç. Şişman and B. Tekin, Phys. Rev. D83 (2011) 061502(R).

[20] H. Lü, Y. Pang and C. N. Pope, Phys. Rev. D84 (2011) 064001.

[21] T. Ç. Şişman, İ. Güllü and B. Tekin, Class. Quant. Grav. 28 (2011) 195004.

[22] N. Ohta, Class. Quant. Grav. 29 (2012) 015002.

[23] N. Kan, K. Kobayashi and Kiyoshi Shiraishi, ISRN Mathematical Physics 2013 (2013) 651684 (9 pages).

[24] N. H. Barth and S. M. Christensen, Phys. Rev. D28 (1983) 1876.

[25] M. Alishahiha and R. Fareghbal, Phys. Rev. D83 (2011) 084052.

[26] E. A. Bergshoeff, O. Hohm, J. Rosseel and P. K. Townsend, Phys. Rev. D83 (2011) 104038.

[27] N. Johansson, A. Naseh and T. Zojer, JHEP 1209 (2012) 114.

[28] D. Grumiller and N. Johansson JHEP 0807 (2008) 134.

[29] D. Grumiller, W. Riedler, J. Rosseel and T. Zojer, arXiv 1302.0280 [hep-th].

[30] E. A. Bergshoeff, S. de Haan, W. Merbis, M. Porrati and J. Rosseel, JHEP 1204 (2012) 134.

[31] E. A. Bergshoeff, S. de Haan, W. Merbis, J. Rosseel and T. Zojer, Phys. Rev. D86 (2012) 064037.

[32] L. Apolo and M. Porrati, JHEP 1208 (2012) 051.

[33] T. Nutma, Phys. Rev. D85 (2012) 124040.

[34] A. Kleinschmidt, T. Nutma and A. Virmani, Gen. Rel. Grav. 45 (2013) 727.

[35] P. Hořava, Phys. Rev. D79 (2009) 084008.

[36] L. Randall and R. Sundrum, Phys. Rev. Lett. 83 (1999) 3370. 
[37] L. Randall and R. Sundrum, Phys. Rev. Lett. 83 (1999) 4690.

[38] U. Camara dS and G. M. Sotkov, Phys. Lett. B694 (2010) 94.

[39] Y.-X. Liu, Y.-Q. Wang, S.-F. Wu and Y. Zhong arXiv:1201.5922 [hep-th]. 